\title{
Modeling of long range frequency sweeping for energetic particle modes
}

\author{
R. M. Nyqvist ${ }^{1, a)}$ and B. N. Breizman ${ }^{2}$ \\ ${ }^{1}$ Department of Earth and Space Sciences, Chalmers University of Technology, SE-412 96 Göteborg, Sweden \\ ${ }^{2}$ Institute for Fusion Studies, The University of Texas at Austin, Austin, Texas 78712, USA
}

(Received 5 March 2013; accepted 20 March 2013; published online 10 April 2013)

\begin{abstract}
Long range frequency sweeping events are simulated numerically within a one-dimensional, electrostatic bump-on-tail model with fast particle sources and collisions. The numerical solution accounts for fast particle trapping and detrapping in an evolving wave field with a fixed wavelength, and it includes three distinct collisions operators: Drag (dynamical friction on the background electrons), Krook-type collisions, and velocity space diffusion. The effects of particle trapping and diffusion on the evolution of holes and clumps are investigated, and the occurrence of nonmonotonic (hooked) frequency sweeping and asymptotically steady holes is discussed. The presented solution constitutes a step towards predictive modeling of frequency sweeping events in more realistic geometries. (C) 2013 American Institute of Physics. [http://dx.doi.org/10.1063/1.4799781]
\end{abstract}

\section{INTRODUCTION}

Frequency sweeping events are common features of experimental plasmas. For instance, the nonlinear evolution of the hot electron interchange instability renders long range frequency sweeping in the Collisionless Terrella Experiment, ${ }^{1}$ and frequency sweeping of toroidal Alfvén eigenmodes (TAEs), fishbone-like energetic particle modes, and compressional and global Alfvén modes have been reported in the National Spherical Tokamak Experiment (NSTX). ${ }^{2,3}$ Likewise, on the Mega Ampère Spherical Tokamak, TAEs driven unstable by energetic particles generated via neutral beam injection often exhibit a bursting evolution of the mode amplitude and a sweep in the mode frequency. ${ }^{4,5}$

Frequency sweeping can be attributed to the evolution of coherent structures, holes and clumps, in the fast particle distribution function. ${ }^{6-9}$ E.g., in a one-dimensional (1D) bump-on-tail model, holes and clumps may form from a marginally unstable plasma mode as a result of an interplay between phase mixing in the wave field and collisional relaxation of the fast particle distribution function. The ensuing frequency sweep is due to the dissipation in the thermal background plasma, which forces the holes and clumps to traverse fast particle phase space and the wave phase velocity to evolve in time.

Sweeping activity is often associated with anomalous transport and redistribution of fast particles. ${ }^{10}$ In particular, long range frequency sweeping has the capacity to convect particles over extended regions of phase space, and it was recently shown that continuous production of holes and clumps at a single resonance may lead to a global relaxation of the fast particle distribution function. ${ }^{11}$ During such long range sweeping events, however, an important, but often overlooked, factor is the slow evolution of the wave field itself. For example, as the carrier frequency of a wave with a fixed spatial period shifts from the original resonance, a gradual deviation from the sinusoidal spatial profile of the linear instability and a corresponding slow change in the

\footnotetext{
a) robert.nyqvist@chalmers.se
}

wave amplitude is enforced through the periodicity requirements on the mode, while fast particle collisions and sources modify the hole/clump structures in phase space. Moreover, as the holes and clumps are conveyed through extended regions of phase space, any detailed study needs to account for the corresponding variation of the equilibrium plasma parameters.

Some important features of long range sweeping have been addressed in previous investigations, cf., Refs. 12 and 13. The former report pinpointed the importance of the mode structure dependence on the frequency shift from the linear resonance. An analytical solution to the 1D bump-on-tail Poisson-Vlasov system was presented and used to describe slowly evolving phase space clumps in the absence of fast particle collisions and sources. In Ref. 13, the analytical solution was generalized to include two types of simplified collision operators (drag and Krook) and allow for slow variations in the equilibrium plasma parameters. In particular, by accounting for a non-constant slope in the fast particle distribution function, the extended model was able to evolve both holes and clumps. It is essential, however, that the holes and clumps in Refs. 12 and 13 are constrained to be of tophat (or waterbag) type. Such fast particle distribution functions have uniform, but evolving, depth/height inside the separatrix, but remain effectively unperturbed outside the trapping area. While the top-hat assumption enables an analytical solution of the problem, it generally breaks down when the evolving wave is allowed to capture new particles. For this reason, the solutions obtained in Refs. 12 and 13 are valid only in the absence of velocity space diffusion (which transports particles through the separatrix) and when the trapping region does not grow.

In the present paper, we relax the previous top-hat constraint and present a self-consistent description of frequency sweeping based on a numerical solution for the evolving hole/clump profile in fast particle phase space. This approach covers particle trapping and detrapping in the wave field due to separatrix expansion/shrinkage and velocity space diffusion of fast particles. The presented work builds on the formalism developed in Ref. 13. As such, it contains not 
only diffusive collisions, but also drag and Krook collision operators, as well as the effects of slow variations in the equilibrium plasma parameters. The paper is organized as follows: Model equations are presented in Sec. II, and the numerical scheme used to evolve the system is outlined in Sec. III. In Sec. IV, we discuss the effects of particle trapping, velocity space diffusion and drag collisions on the mode evolution and frequency shift, including features such as non-monotonic sweeping patterns and the formation of asymptotically steady holes. Steady state holes are then further analyzed in Sec. V. Finally, Sec. VI concludes the presented work.

\section{ADIABATIC MODEL (BASIC EQUATIONS)}

As in Ref. 13, we employ a 1D bump-on-tail model to describe the evolution of phase space holes and clumps. We limit our investigation to the so called adiabatic regime, in which a single perturbation (with fixed spatial period $\lambda$ ) evolves slowly compared to the fast motion of particles resonant with the wave. Formally, the appropriate ordering is given by

$$
\left[\frac{d \ln \omega_{B}}{d t}, \frac{d \ln \dot{s}}{d t}\right] \ll \omega_{B} \sim \gamma_{L} \sim \gamma_{d} \ll \omega \sim \omega_{0},
$$

where the bounce frequency $\omega_{B}$ of particles trapped in the wave field serves as a measure of the wave amplitude, $\dot{s}$ is the wave phase velocity, and $\omega=k \dot{s}$ the corresponding wave carrier frequency (with $k=2 \pi / \lambda$ the wave number). The quantities $\gamma_{L}$ and $\gamma_{d}$ denote, respectively, the linear growth rate due to the positive slope of the unperturbed energetic particle equilibrium distribution and the rate of damping due to dissipation in the background plasma. The adiabatic ordering (1) is designed to describe well defined spectral lines that extend over frequency ranges comparable to the resonant frequency $\omega_{0}=k \dot{s}_{0}$.

The relevant adiabatic equations were derived in Ref. 13. They are to be solved self-consistently for the following three quantities: 1) the wave field potential $\mathcal{U}(\xi ; \tau)$, which is periodic in the wave frame spatial variable $\xi \in[0,1]$ and a slow function of normalized time,

$$
\tau=\frac{\pi}{2}\left(\frac{16}{3 \pi^{2}}\right)^{3}\left(\frac{\gamma_{L 0}}{\omega_{0}}\right)^{2} \gamma_{L 0} t
$$

as compared to the fast resonant particle motion; 2) the perturbed distribution of trapped particles, $\delta \mathcal{F}(\mathcal{J} ; \tau)$, which evolves slowly with $\tau$ and whose phase space profile depends on the action variable (adiabatic invariant of the trapped particle motion)

$$
\mathcal{J}(\mathcal{E} ; \tau)=\frac{\pi}{2 \sqrt{2}} \int_{\xi_{*}}^{1-\xi_{*}} \sqrt{\mathcal{E}-\mathcal{U}(\xi ; \tau)} d \xi,
$$

with $\mathcal{E}=u^{2} / 2+\mathcal{U}(\xi ; \tau)$ the fast particle energy in the wave frame and $u$ the corresponding velocity; 3 ) the wave phase velocity $\dot{s}(\tau)$. The system of equations ${ }^{13}$ contains a kinetic equation for the trapped particles

$$
\begin{aligned}
\frac{\partial \delta \mathcal{F}}{\partial \tau}+\tilde{\beta} \delta \mathcal{F}= & -\rho\left[\frac{d}{d \tau}\left(\frac{\dot{s}}{\dot{s}_{0}}\right)+\tilde{\alpha}^{2}\right] \\
& +\tilde{\nu}^{3} \frac{\partial}{\partial \mathcal{J}}\left[\mathcal{J} \frac{\partial \mathcal{J}}{\partial \mathcal{E}} \frac{\partial \delta \mathcal{F}}{\partial \mathcal{J}}\right]
\end{aligned}
$$

a Poisson equation

$$
\begin{aligned}
& \frac{\partial^{2} \mathcal{U}}{\partial \xi^{2}}+4 \pi^{2}\left(\frac{\dot{s}_{0}}{\dot{s}}\right)^{2} \mathcal{U} \\
& \quad=3 \sqrt{2} \pi^{3}\left[\int_{\mathcal{J}(\mathcal{E}=\mathcal{U})}^{\mathcal{J} S} \sqrt{\mathcal{E}(\mathcal{J})-\mathcal{U}} \frac{\partial \delta \mathcal{F}}{\partial \mathcal{J}} d \mathcal{J}-C_{1}\right]
\end{aligned}
$$

with

$$
C_{1}(\tau)=\int_{0}^{1} \int_{\mathcal{J}(\mathcal{E}=\mathcal{U})}^{\mathcal{J}_{S}} \sqrt{\mathcal{E}(\mathcal{J} ; \tau)-\mathcal{U}(\xi ; \tau)} \frac{\partial \delta \mathcal{F}}{\partial \mathcal{J}} d \mathcal{J} d \xi,
$$

and a power balance condition,

$$
\frac{d}{d \tau}\left(\frac{\dot{s}}{\dot{s_{0}}}\right)+\tilde{\alpha}^{2}=\frac{\gamma_{d}}{\gamma_{L 0}} \frac{1}{\sqrt{2} C_{1}}\left(\frac{\dot{s}_{0}}{\dot{s}}\right)^{3} \int_{0}^{1}(\mathcal{U}(\xi))^{2} d \xi
$$

that equates the energy released by fast particles during frequency sweeping to the dissipation in the background plasma. In these equations, $\tilde{\alpha}, \tilde{\beta}$, and $\tilde{\nu}$ are normalized collision frequencies for the simplified drag, Krook, and velocity space diffusion collision operators (for the sake of simplicity, these frequencies are assumed constant throughout this article, although they generally depend on $\dot{s}$ ), and the function $\rho(\dot{s})$ characterizes the local slope of the unperturbed fast particle distribution. The maxima and minimum of the periodic potential $\mathcal{U}$, denoted $\mathcal{U}_{\max }$ and $\mathcal{U}_{\text {min }}$, are located at the boundary $\xi=0,1$ and midpoint $\xi=0.5$, respectively, and the boundary conditions for $\delta \mathcal{F}$ read

$$
\delta \mathcal{F}\left(\mathcal{J}_{S} ; \tau\right)=0,\left.\quad \frac{\partial \delta \mathcal{F}}{\partial \mathcal{J}}\right|_{\mathcal{J}=0}=0
$$

where

$$
\mathcal{J}_{S}(\tau)=\int_{0}^{1} \sqrt{\mathcal{U}_{\max }(\tau)-\mathcal{U}(\xi ; \tau)} d \xi
$$

is the action evaluated at the separatrix orbit with $\mathcal{E}=\mathcal{U}_{\max }$. The turning points $\xi_{*}$ and $1-\xi_{*}$ for trapped particles with energy $\mathcal{E}$ are given by $\mathcal{U}\left(\xi_{*}\right)=\mathcal{E}$. The relation $\mathcal{E}=\mathcal{E}(\mathcal{J} ; \tau)$ is defined implicitly through Eq. (3). Finally, all quantities denoted with a subscript 0 represent initial values at $\tau=0$. The reader is referred to Ref. 13 for normalizations and additional details.

The adiabatic ordering (1) breaks down for particles near the separatrix, at which the bounce period diverges logarithmically and particles formally come to a halt at the turning points $\xi=0,1$. In reality, however, particles do not linger at the separatrix orbit. Instead, they either cross the separatrix as the mode structure evolves or due to diffusive collisions or skim the trapping region as a result of frequency 
sweeping or drag. In any case, the adiabatic invariants of particles with kinetic energies in a thin transition layer centered at the separatrix experience phase dependent jumps. ${ }^{14,15}$ It can be shown, however, that due to the Liouville theorem, it is possible to describe the integrated effect of these jumps via proper relabeling of the particle orbits. ${ }^{16}$ For a uniform distribution of ambient particles, particle trapping/detrapping can therefore be modeled, as in Eq. (4a), by simply including/ excluding, to/from the trapping area, thin annuli of phase space on which the adiabatic invariant and particle distribution function are constant.

With regards to numerical modeling, a slightly inconvenient aspect of the separatrix evolution is that the upper boundary of the trapped particle domain $\left[0, \mathcal{J}_{S}(\tau)\right]$ evolves in time with the mode structure and amplitude, as seen in Eq. (6). An apparent remedy is to change variable from $\mathcal{J}$ to

$$
E(\mathcal{J} ; \tau) \equiv \frac{\mathcal{E}(\mathcal{J} ; \tau)-\mathcal{U}_{\min }(\tau)}{2 a(\tau)}
$$

where

$$
a(\tau) \equiv \frac{\mathcal{U}_{\max }(\tau)-\mathcal{U}_{\min }(\tau)}{2}
$$

is a measure of the wave amplitude. If we transform the potential similarly to

$$
y(\xi ; \tau) \equiv \frac{\mathcal{U}(\xi ; \tau)-\mathcal{U}_{\min }(\tau)}{2 a(\tau)},
$$

both $E$ and $y$ will be restricted to $[0,1]$ at all times, while Eqs. (4a), (4b), and (4d) transform into

$$
\begin{gathered}
\frac{\partial \delta \mathcal{F}}{\partial \tau}=\left[\frac{\tilde{\nu}^{3}}{2 a}-\frac{d E}{d \tau}\right] \frac{\partial \delta \mathcal{F}}{\partial E}+\frac{\tilde{\nu}^{3}}{a} G \frac{\partial^{2} \delta \mathcal{F}}{\partial E^{2}}-\rho\left[\frac{d}{d \tau}\left(\frac{\dot{s}}{\dot{s}_{0}}\right)+\tilde{\alpha}^{2}\right], \\
\frac{\partial^{2} y}{\partial \xi^{2}}+4 \pi^{2}\left(\frac{\dot{s}_{0}}{\dot{s}}\right)^{2} y=\frac{3 \pi^{3}}{\sqrt{a}}\left[\int_{y}^{1} \sqrt{E-y} \frac{\partial \delta \mathcal{F}}{\partial E} d E-C_{2}\right],
\end{gathered}
$$

and

$$
\frac{d}{d \tau}\left(\frac{\dot{s}}{\dot{s}_{0}}\right)+\tilde{\alpha}^{2}=\frac{\gamma_{d}}{\gamma_{L 0}} \frac{1}{\sqrt{2} C_{1}}\left(\frac{\dot{s}_{0}}{\dot{s}}\right)^{3} \int_{0}^{1}\left[2 a y(\xi)+\mathcal{U}_{\min }\right]^{2} d \xi .
$$

In these equations

$$
\begin{gathered}
G(E ; \tau)=\int_{\xi_{*}}^{1-\xi_{*}} \sqrt{E-y(\xi ; \tau)} d \xi\left[\int_{\xi_{*}}^{1-\xi_{*}} \frac{d \xi}{\sqrt{E-y(\xi ; \tau)}}\right]^{-1}, \\
C_{2}(\tau)=\frac{C_{1}}{\sqrt{2 a}}+\frac{2}{3 \pi} \frac{\mathcal{U}_{\min }}{\sqrt{a}}\left(\frac{\dot{s}_{0}}{\dot{s}}\right)^{2}
\end{gathered}
$$

and $\mathcal{U}_{\min }$ is given by the condition that $\mathcal{U}$ averages to zero over the wave spatial period. This constraint ensures that there is no average space charge in the plasma. Consequently, integration of Eq. (9) gives

$$
\mathcal{U}_{\min }(\tau)=-2 a(\tau) \int_{0}^{1} y(\xi ; \tau) d \xi
$$

The time derivative of the normalized energy, $d E / d \tau$, is determined through the invariance of $\mathcal{J}=\mathcal{J}(\mathcal{E}(\tau) ; \tau)$,

$$
\frac{d \mathcal{J}}{d \tau}=\frac{\partial \mathcal{J}}{\partial \mathcal{E}} \frac{d \mathcal{E}}{d \tau}+\frac{\partial \mathcal{J}}{\partial \tau}=0
$$

giving

$$
\frac{d \mathcal{E}}{d \tau}=-\frac{\partial \mathcal{J}}{\partial \tau}\left(\frac{\partial \mathcal{J}}{\partial \mathcal{E}}\right)^{-1}
$$

In terms of $E, y$, and $a$, we get

$$
\begin{aligned}
\frac{d E}{d \tau}= & \left\{\int_{\xi_{*}}^{1-\xi_{*}} \frac{\partial y / \partial \tau}{\sqrt{E-y(\xi ; \tau)}} d \xi-\frac{1}{a} \frac{d a}{d \tau} \int_{\xi_{*}}^{1-\xi_{*}} \sqrt{E-y(\xi ; \tau)} d \xi\right\} \\
& \times\left\{\int_{\xi_{*}}^{1-\xi_{*}} \frac{d \xi}{\sqrt{E-y(\xi ; \tau)}}\right\}
\end{aligned}
$$

We have deliberately dropped the Krook term in Eq. (10a) by setting $\tilde{\beta}=0$. Since the effect of Krook-type collisions was thoroughly analyzed in Ref. 13, the present investigation is devoted to the (more complicated) study of the more realistic diffusion operator.

The transformations (7) and (9) also provide a handy formula for $C_{2}$ in terms of $a, \delta \mathcal{F}$, and $\dot{s}$. To see this, we first multiply Eq. (10b) by $\partial y / \partial \xi$ and integrate from 0 to $\xi$. Incorporating the boundary conditions $y=1$ and $\partial y / \partial \xi=0$ at $\xi=0$, we obtain

$$
\begin{aligned}
& \frac{1}{2}\left(\frac{\partial y}{\partial \xi}\right)^{2}+2 \pi^{2}\left(\frac{\dot{s}_{0}}{\dot{s}}\right)^{2}\left(y^{2}-1\right) \\
& \quad=\frac{3 \pi^{3}}{\sqrt{a}} \times\left\{C_{2}(1-y)-\int_{y(\xi)}^{1} \int_{x}^{1} \sqrt{E-x} \frac{\partial \delta \mathcal{F}}{\partial E} d E d x\right\} .
\end{aligned}
$$

Evaluating Eq. (17) at $\xi=0.5$, where $y=\partial y / \partial \xi=0$, we find

$$
C_{2}=\int_{0}^{1} \int_{x}^{1} \sqrt{E-x} \frac{\partial \delta \mathcal{F}}{\partial E} d E d x-\frac{2 \sqrt{a}}{3 \pi}\left(\frac{\dot{s}_{0}}{\dot{s}}\right)^{2} .
$$

The integral in Eq. (18) is easy to evaluate numerically.

\section{NUMERICAL MODELLING}

We split the discussion of numerics into two parts, presented in Subsections III A and IIIB. Subsection III A 
focuses on and motivates the initialization of the system. Subsection III B describes the numerical scheme used to advance the equations.

\section{A. System initialization}

Proper initialization of phase space structures is essential for the adiabatic description, since spontaneous formation of holes and clumps from a weakly unstable linear wave involves an intrinsically non-adiabatic explosive growth. ${ }^{6,17}$ The system enters the adiabatic phase only after the explosive growth has terminated, due to phase mixing of the trapped particles, and the hole/clump formation has been completed. Then, during the early adiabatic phase (but for $\tau$ larger than the hole/clump formation time), the frequency sweep is well described by the square root scaling $\left(\dot{s}-\dot{s}_{0}\right) / \dot{s}_{0} \propto \sqrt{\tau}$, first derived in Ref. 6. A more formal reason to exclude small values of $\tau$ from the adiabatic modeling is that the square root scaling renders $d \ln \dot{s} / d \tau \propto 1 / \sqrt{\tau}$, which obviously violates the adiabatic ordering (1) as $\tau \rightarrow 0$. We therefore start our simulations with a prescribed hole/ clump structure at some small but finite time $\tau_{i}$, with corresponding wave phase velocity $\dot{s}_{i} \equiv \dot{s}\left(\tau_{i}\right)$ satisfying $\left(\dot{s}_{i}-\dot{s}_{0}\right) / \dot{s}_{0} \propto \sqrt{\tau_{i}}$. The form we choose for the initial trapped particle perturbed distribution, $\delta \mathcal{F}\left(E ; \tau_{i}\right)$, is a generalization of the previously used discontinuous top-hat distribution. It now includes a finite-width transition layer with an adjustable exponential profile that extends inwards from the separatrix in $E$-space,

$$
\delta \mathcal{F}\left(E ; \tau_{i}\right)=\frac{\dot{s}_{0}-\dot{s}_{i}}{\dot{s}_{0}}\left[1-\exp \left(\frac{E-1}{w_{i}}\right)\right] .
$$

The first factor on the right hand side of Eq. (19) takes into account that the hole/clump depth/height is initially proportional to the shift in phase velocity from that of the seed linear wave. One can show ${ }^{13}$ that to lowest order in $\left(\dot{s}-\dot{s}_{0}\right) / \dot{s}_{0}$, collisions are unimportant, and the equilibrium distribution can be regarded as linear, $\rho(\dot{s}) \approx 1$. Neglecting separatrix expansion, the kinetic equation (4a) can then be immediately integrated to give the uniform level $\delta \mathcal{F} \approx\left(\dot{s}_{0}-\dot{s}\right) / \dot{s}_{0}$. The second factor in Eq. (19) describes the profile of the initial distribution in $E$-space, with the adjustable parameter $w_{i}$ characterizing its gradient. Plots of $\delta \mathcal{F}\left(E ; \tau_{i}\right)$ for a hole with $w_{i}$ ranging from 0.05 to 0.25 are shown in Figure 1, and Figure 2 displays the corresponding structure in $(\xi, u)$-phase space when $w_{i}=0.292$ (this particular value of $w_{i}$ will be used extensively in the simulations in Sec. IV). While the choice of $E$-space profile in Eq. (19) may seem arbitrary, it reflects the observation that frequency sweeping in the adiabatic regime tends to maintain a discontinuous derivative at the separatrix. Moreover, Eq. (19) closely replicates the type of structures that rapidly emerge from other initial profiles. In particular, an initial Gaussian profile centered at $E=1$ relaxes to a shape very similar to Eq. (19) within a few iterations.

From the Poisson equation (10b), we expect the mode amplitude to vary with $\delta \mathcal{F}$. The initial mode amplitude

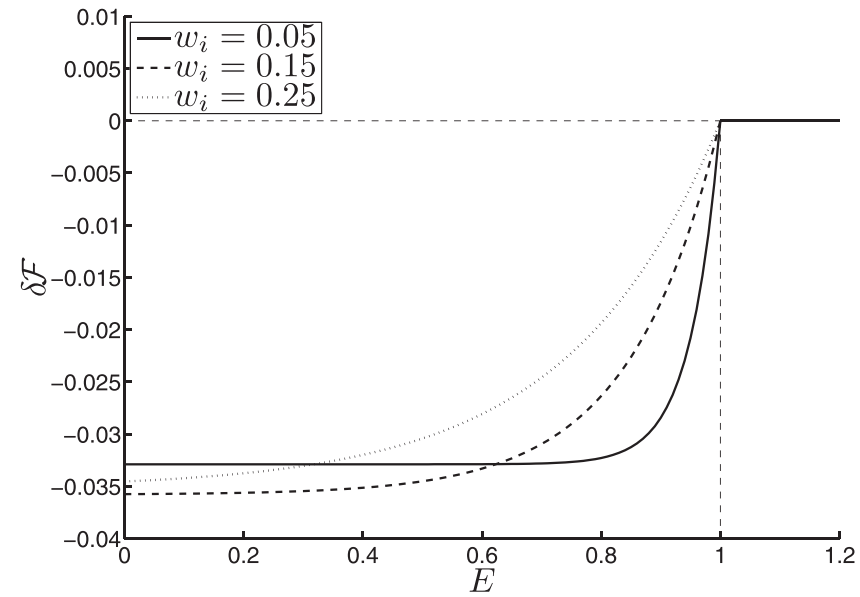

FIG. 1. Initial perturbed fast particle distributions, $\delta \mathcal{F}\left(E ; \tau_{i}\right)$, for values of $w_{i}$ ranging from 0.05 to 0.25 .

should therefore depend on $w_{i}$. This dependence can be obtained from the following approximate relation: ${ }^{13}$

$$
\sqrt{a} \approx-3 \pi \frac{\dot{s}^{2}}{\dot{s}^{2}-\dot{s}_{0}^{2}} \int_{0}^{1} \cos 2 \pi \xi \int_{y_{0}(\xi)}^{1} \sqrt{E-y_{0}(\xi)} \frac{\partial \delta \mathcal{F}}{\partial E} d E d \xi .
$$

Equation (20) holds for $\left|\dot{s}-\dot{s}_{0}\right| / \dot{s}_{0} \ll 1$, i.e., when the mode structure is nearly sinusoidal, $y(\xi) \approx y_{0}(\xi) \equiv(1+\cos 2 \pi \xi) / 2$. Evaluating Eq. (20) at $\tau=\tau_{i}$ by substituting the distribution (19) and $\dot{s}=\dot{s}_{i}$ and then taking the limit $\tau_{i} \rightarrow 0$ (meaning that $\dot{s}_{i} \rightarrow \dot{s}_{0}$ ), we find

$$
a_{0}=\frac{9 \pi^{2} I_{1}^{2}}{4}
$$

where

$$
I_{1}\left(w_{i}\right) \equiv \frac{1}{w_{i}} \int_{0}^{1} \cos 2 \pi \xi \int_{y_{0}(\xi)}^{1} \sqrt{E-y_{0}(\xi)} \exp \left(\frac{E-1}{w_{i}}\right) d E d \xi .
$$

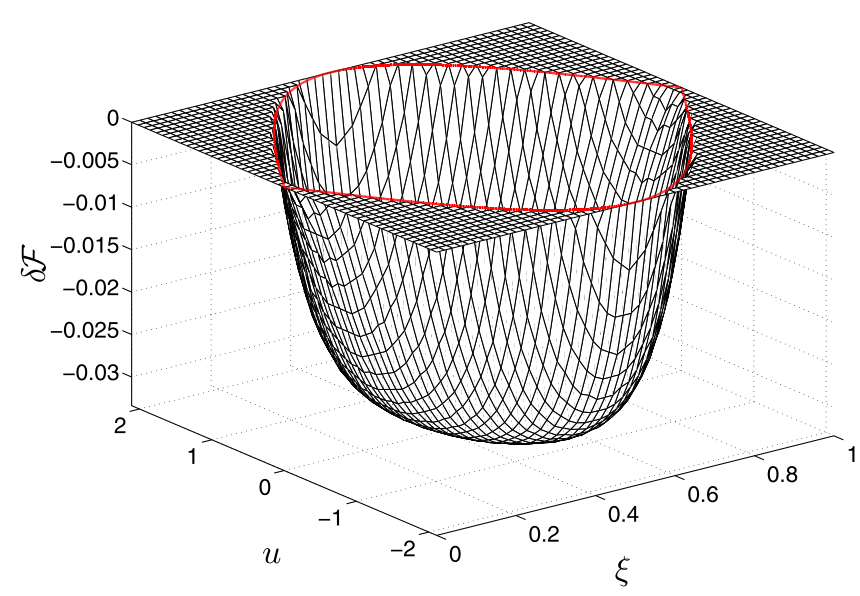

FIG. 2. Initial perturbed fast particle distribution, $\delta \mathcal{F}\left(E ; \tau_{i}\right)$, for $w_{i}=0.292$, plotted as a function of the phase space variables $(\xi, u)$. The separatrix is highlighted by the red curve. 


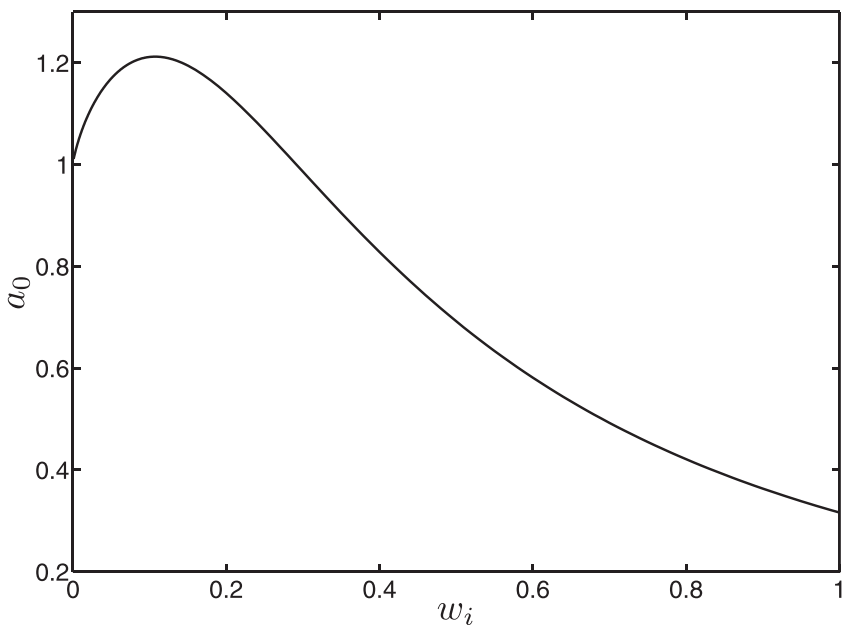

FIG. 3. Plot of the initial mode amplitude $a_{0}$ as a function of $w_{i}$. For large $w_{i}$, the curve tends asymptotically to 0 .

Figure 3 shows that $a_{0}$ is a non-monotonic function of $w_{i}$ : For small $w_{i}, a_{0}$ increases from 1 at $w_{i}=0$, corresponding to the previously discussed top-hat distribution, to its maximum $a_{0}=1.212$ at $w_{i}=0.107$. It then decreases monotonically, passing through unity again at $w_{i}=0.292$ and tending asymptotically to 0 for large $w_{i}$. Thus, a reduction in $\delta \mathcal{F}$ close to the separatrix leads to an increase in the wave amplitude, while a more global reduction that covers a larger part of the trapping area is needed in order to generate an amplitude reduction. This trend was first observed by a different method $^{18}$ and then confirmed ${ }^{13}$ for a step-like (rather than exponential) profile of $\delta \mathcal{F}\left(E ; \tau_{i}\right)$ inside the separatrix.

Substitution of $a_{0}$ and $y_{0}$ in the power balance condition (10c) then gives, to lowest order in $\left(\dot{s}-\dot{s}_{0}\right) / \dot{s}_{0}$, the square root dependence

$$
\dot{s} / \dot{s}_{0}=1 \pm\left[\frac{\gamma_{d}}{\gamma_{L 0}} \frac{a_{0}^{3 / 2}}{2 I_{2}} \tau\right]^{1 / 2}
$$

where

$$
I_{2}\left(w_{i}\right) \equiv \frac{1}{w_{i}} \int_{0}^{1} \int_{y_{0}(\xi)}^{1} \sqrt{E-y_{0}(\xi)} \exp \left(\frac{E-1}{w_{i}}\right) d E d \xi
$$

and the \pm refers to holes and clumps, respectively. We can then evaluate $\dot{s}_{i}\left(w_{i}\right)$ by substituting $\tau_{i}$ and $w_{i}$ in Eq. (23), which completes the system initialization in Eq. (19).

As a final remark, we remind the reader that top-hat holes are only allowed to evolve adiabatically when the slope of the unperturbed fast particle distribution decreases with increasing $\dot{s}$ sufficiently fast (for $\dot{s}>\dot{s}_{0}$ ). ${ }^{13}$ In particular, there exists a critical value

$$
K_{c}=-2\left[4-\left(\frac{\pi}{2}\right)^{2}+\frac{4}{\pi} \frac{\gamma_{L 0}}{\gamma_{d}} \tilde{\alpha}^{2}\right]
$$

of

$$
\left.K \equiv \dot{s}_{0} \frac{\partial \rho}{\partial \dot{s}}\right|_{\dot{s}=\dot{s}_{0}},
$$

such that for $K>K_{c}$, the trapping areas of holes initially grow and those of clumps shrink, whereas for $K<K_{c}$ the opposite holds. For $K=K_{c}$, neither holes nor clumps initially expand. When top-hat holes are simulated in slopes with $K>K_{c}$, the initial separatrix expansion triggers a nonadiabatic growth of the trapping area that results in significant particle trapping. In our case, however, such a sudden transition may be avoided by choosing a sufficiently large value of $w_{i}$ in Eq. (19). This observation further motivates our use of the form (19), where the value of the adjustable parameter $w_{i}$ can be tuned to ensure that the system takes off smoothly.

\section{B. Numerical scheme}

Once the system has been initialized in accordance with Eq. (19), we use the following step-by-step procedure to determine its evolution:

1. Calculate the frequency sweeping rate from the power balance condition (10c) and update the wave phase velocity accordingly.

2. Update the trapped particle perturbed distribution function (hole/clump profile in phase space) at the new phase velocity by solving the kinetic equation (10a).

3. Calculate the wave potential profile $y(\xi)$ and amplitude $a$ by solving the Poisson equation (10b).

4. Repeat from step 1.

A minor point of concern is that Eq. (10a) is solved prior to Eq. (10b), even though Eq. (10a) requires knowledge of the updated $y$ and $a$. This issue is addressed by iterating over steps 2 and 3 a few times during each time step, until the iterations converge.

The Poisson equation is solved by means of a shooting method: Given a guessed value of $a$, we find $C_{2}$ from Eq. (18) and then use Eq. (10b) to calculate $y$ step by step from $\xi=0$ to $\xi=1$. The process is repeated until the resulting profile exhibits a period of 1 , at which point $a$ has been found. A linear interpolation based on the two preceding values provides the input guess for $a$, after which a small interval that brackets the root is created and finally a simple bisection method is used to ensnare the correct value.

The kinetic equation is advanced in time by means of a Crank-Nicholson finite difference scheme that incorporates the boundary conditions (5). Although well suited for the diffusive piece of Eq. (10a), the Crank-Nicholson method is not optimal for the description of advection. Being a central scheme, it generates spurious oscillations when used to advect sharp edges. In our case a sharp edge indeed arises at the separatrix due to the frequency sweeping or as a result of a shrinking trapping region. The usual remedy is to introduce a small amount of artificial, numerical diffusion by adopting an upwind difference scheme. The present model already comprises diffusion, however, so it suffices to make sure that the diffusivity is always large enough to ensure smooth wave evolution. Moreover, oscillations during the early evolution 
stages are avoided by setting up holes and clumps with somewhat relaxed gradients $\partial \delta \mathcal{F} / \partial E$, i.e., large enough $w_{i}$. As will be discussed in Sec. IV A, most of our simulations will have $w_{i}=0.292$, a value at which no initial oscillations appear.

We conclude this section with a remark about the terms $d E / d \tau$ and $G(E ; \tau)$ that appear in Eq. (10a). For $E \ll 1$, direct numerical evaluation of Eqs. (16) and (11) sometimes generates relatively large errors, arising from numerical inaccuracies in $y(\xi)$ around its minimum at $\xi=0.5$. These errors are eliminated by expanding $y(\xi)$ around $\xi=0.5$ to second order and evaluating the integrals in $G(E ; \tau)$ and $d E / d \tau$ analytically for $0<E \ll 1$. Moreover, as some of the integrands in Eqs. (16) and (11) are singular at $\xi_{*}$ and $1-\xi_{*}$, we evaluate the turning point contributions to the integrals analytically for small values of $E-y(\xi)$.

\section{SIMULATION RESULTS}

In this section, we use a MATLAB implementation of the numerical model in Sec. III to examine the effects of particle trapping and detrapping on frequency sweeping scenarios. The evolution of the system is controlled by five input variables:

1. The slope of the unperturbed fast particle distribution, $\rho(\dot{s})$.

2. The rate of drag, $\tilde{\alpha}$.

3. The rate of diffusive collisions, $\tilde{\nu}$.

4. The initial hole/clump profile in $E$-space, set by $w_{i}$.

5. The ratio $\gamma_{d} / \gamma_{L 0}$, which regulates the frequency sweeping rate through Eq. (10c).

In what follows, all simulations are performed with $\gamma_{d} / \gamma_{L 0}=1$ and $w_{i}=0.292$ (except for the runs presented in Figure 8). We first investigate the effect of particle trapping due to separatrix expansion in Sec. IV A. These simulations are performed with $\tilde{\alpha}=\tilde{\nu}=0$, so that no particles enter the trapping area due to diffusive collisions, and separatrix expansion is enforced by adopting a constant slope in the unperturbed fast particle distribution, $\rho(\dot{s})=1$, with $K=0>K_{c}$. In fact, for $\dot{s}>\dot{s}_{0}$, the constant slope is everywhere greater than the critical slope $e^{13}$

$$
\rho_{c}(\dot{s})=\frac{6}{\pi\left(\dot{s} / \dot{s}_{0}\right)^{4}}[\eta-\tan \eta]^{-1}+\frac{\tan ^{2} \eta}{\left(\dot{s} / \dot{s}_{0}\right)^{5}}[\eta-\tan \eta]^{-2},
$$

where $\eta \equiv \pi \dot{s}_{0} / 2 \dot{s}$. The critical slope has $K=K_{c}$ and is designed so that top-hat structures evolve with a constant trapping area, $\mathcal{J}_{S}=1$, when $\tilde{\alpha}=\tilde{\nu}=0$. The corresponding critical equilibrium distribution is given by

$$
\mathcal{F}_{0 c}(\dot{s})=\mathcal{F}_{0}\left(\dot{s}_{0}\right)+\frac{2}{\pi}\left(\frac{\dot{s}_{0}}{\dot{s}}\right)^{3}[\tan \eta-\eta]^{-1} .
$$

The constant slope is therefore expected to yield an expanding hole separatrix and increasing wave amplitude. We then address hole/clump evolution in the presence of fast particle collisions. In Sec. IV B, we investigate the impact of velocity space diffusion on the lifetimes of holes and clumps. Finally, Sec. IV C deals with non-monotonic frequency sweeping,

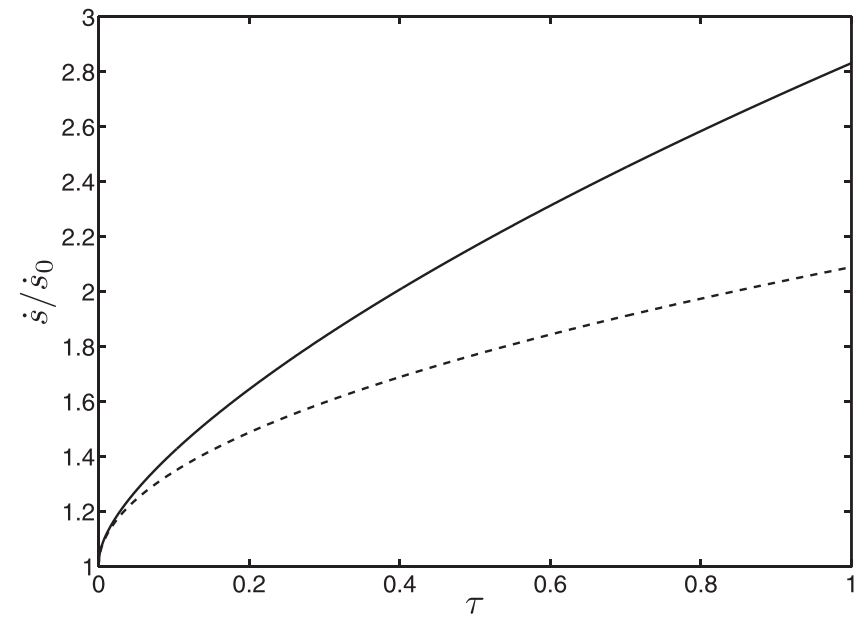

FIG. 4. Frequency evolution of hole with a linear equilibrium distribution profile, $\rho(\dot{s})=1$, and $\tilde{\alpha}=\tilde{\beta}=\tilde{\nu}=0$. The solid line is the simulation result and the dashed line, included here for comparison, is the square root scaling (23).

which may arise when $\tilde{\alpha} \neq 0$. We discuss when sweep reversal occurs and map domains with different types of mode evolution onto the $(\tilde{\alpha}, \tilde{\nu})$-plane.

\section{A. Effect of separatrix expansion}

As previously discussed in Sec. II, the inclusion of ambient particles via a small expansion of the separatrix mainly affects the trapped particle area in the vicinity of the separatrix. As the wave amplitude increases, the particles enter a narrow layer just inside the separatrix on which the perturbed distribution $\delta \mathcal{F}$ momentarily vanishes. If the expansion continues, slowly enough to preserve adiabaticity, the process results in a reduction of $\partial \delta \mathcal{F} / \partial E$ that gradually spreads inwards. The long term effect of particle trapping due to separatrix expansion is shown in Figures 4-6, which display the evolution of $\dot{s}, a$, and $\delta \mathcal{F}$ for a phase space hole simulated with a constant slope of the equilibrium distribution function, $\rho(\dot{s})=1$, and $\tilde{\alpha}=\tilde{\nu}=0$. Figures 4 and 5 show how the growing wave amplitude boosts the frequency sweeping rate through Eq. (10c). The increased rate of sweeping then

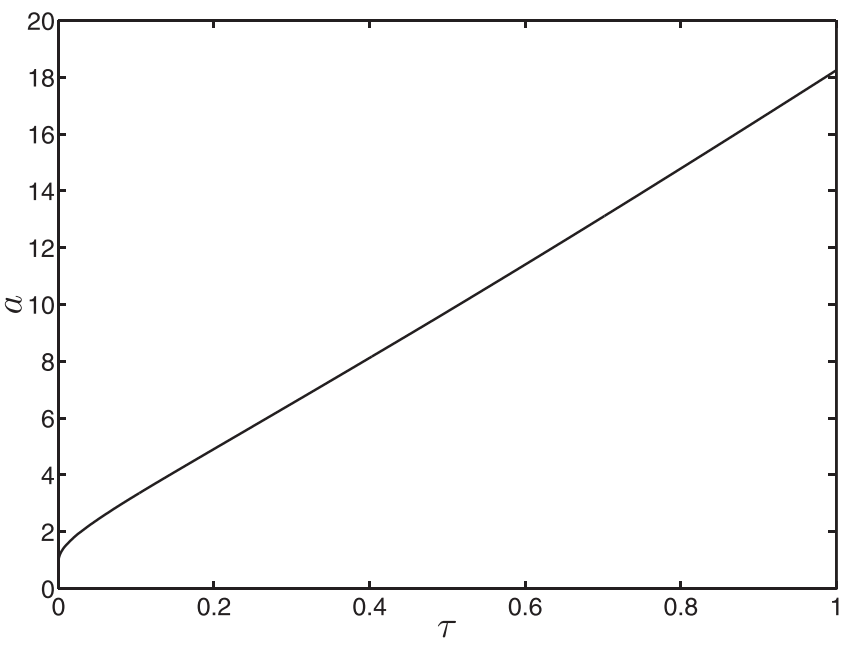

FIG. 5. Amplitude evolution corresponding to the spectrogram in Figure 4. 


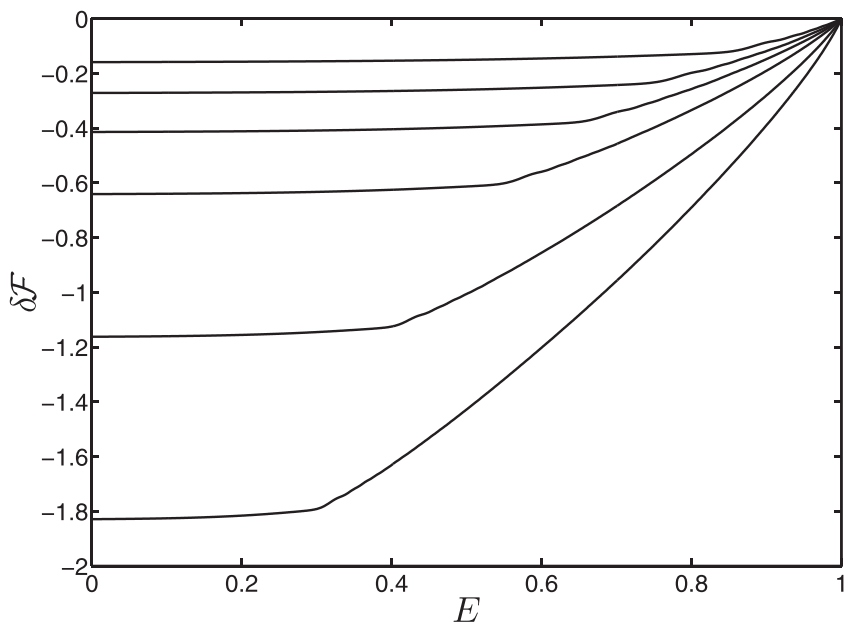

FIG. 6. Snapshots of $\delta \mathcal{F}(E ; \tau)$ for the hole evolution in Figures 4 and 5. The curves correspond to, from top to bottom, $\tau=0.02,0.05,0.1,0.2,0.5,1$.

further elevates the amplitude growth in a feedback-like manner as the hole deepens, cf., Figures 6 and 7. As a result, the amplitude increases at a roughly steady rate. The particle trapping progresses as expected, and the overall frequency sweep is considerably faster than the square root scaling (23), given by the dashed line in Figure 4.

The choice $w_{i}=0.292$ can be motivated by looking more carefully at the amplitude evolution in Figure 5 for small values of $\tau$. Figure 8 shows the initial amplitude evolution, $0 \leq \tau \leq 0.03$, for a series of holes initialized with $w_{i}=0.02,0.107,0.2,0.292$, and 0.4. These values are chosen so that $w_{i}=0.02$ is to the left of the maximum of $a_{0}$ in Figure 3, $w_{i}=0.107$ corresponds to the maximum, and $w_{i}=0.2$ is between the maximum and $w_{i}=0.292$, where $a_{0}$ equals unity. The largest value, $w_{i}=0.4$, represents a hole with $a_{0}<1$. In general, the initial separatrix expansion of holes in slopes with $K>K_{c}$ always induces particle trapping, but the effect of the induced particle trapping varies depending on $w_{i}$. For $w_{i} \lesssim 0.107$, Figure 3 suggests that the particle trapping should result in even further amplitude growth. This expectation is confirmed by the initial fast and irregular increase of $a$ exhibited by the $w_{i}=0.02$-curve in Figure 8 . For $w_{i} \gtrsim 0.107$, however, the trapping should

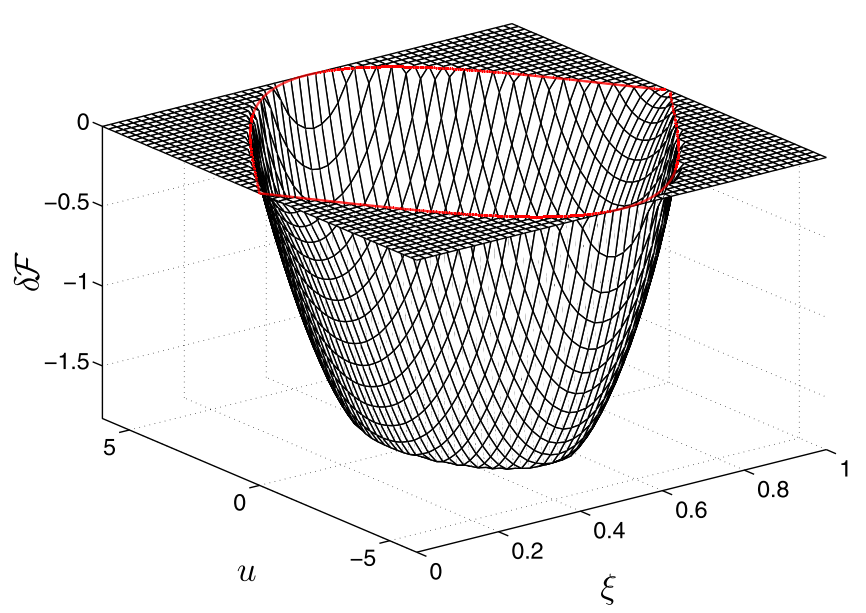

FIG. 7. Phase space plot of $\delta \mathcal{F}$ for the hole at $\tau=1$ in Figure 6.

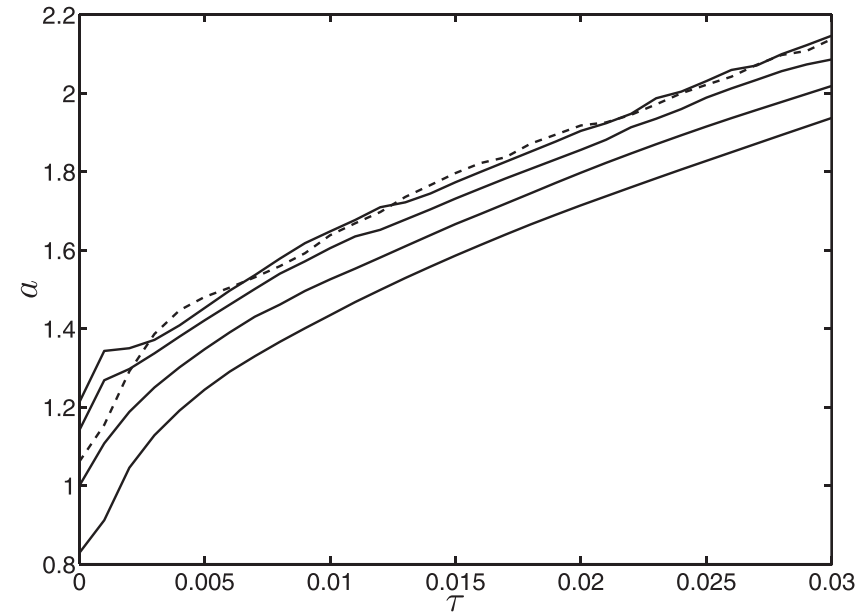

FIG. 8. Initial amplitude evolution for holes with varying $w_{i}$, simulated with a constant slope. The dashed line has $w_{i}=0.02$, and the solid lines have, from top to bottom, $w_{i}=0.107,0.2,0.292$, and 0.4 .

somewhat compensate for the original amplitude increase associated with $K>K_{c}$. This trend is also observed in Figure 8. At $w_{i}=0.292$, we find the smoothest possible take-off. Then, for $w_{i}>0.292$, the induced particle trapping decreases the amplitude too much, so that its initial evolution is again irregular. Finally, we note that all the initial irregularities in Figure 8 fade away with time and that the subsequent evolution is in fact smooth regardless of the initial evolution.

\section{B. Effect of diffusion}

Velocity space diffusion tends to suppress frequency sweeping by filling up holes and reducing clumps. This effect is shown in Figures 9-11, where the solid, dashed, and dotted lines correspond to $\tilde{\nu}=1.0,1.2$, and 1.4, respectively. For both holes and clumps, the frequency sweeps in Figure 9 progress at a slower rate and terminate earlier with increasing $\tilde{\nu}$, which is also reflected in the amplitude plots in Figures 10 and 11. In order to isolate the effect of diffusion, we use the critical slope (27) in the simulations of holes, thereby minimizing the effect of particle trapping due to

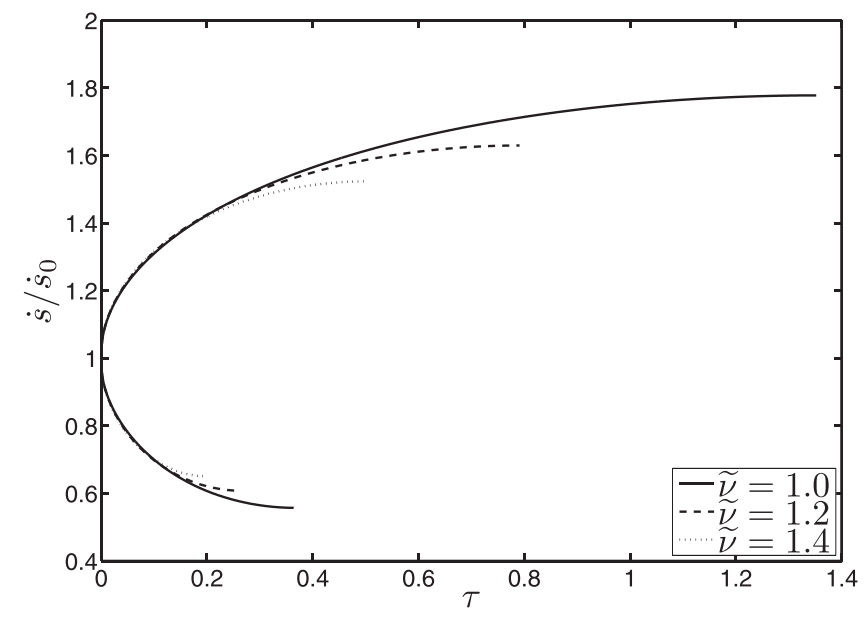

FIG. 9. Frequency evolution of holes and clumps in the absence of drag collisions. 


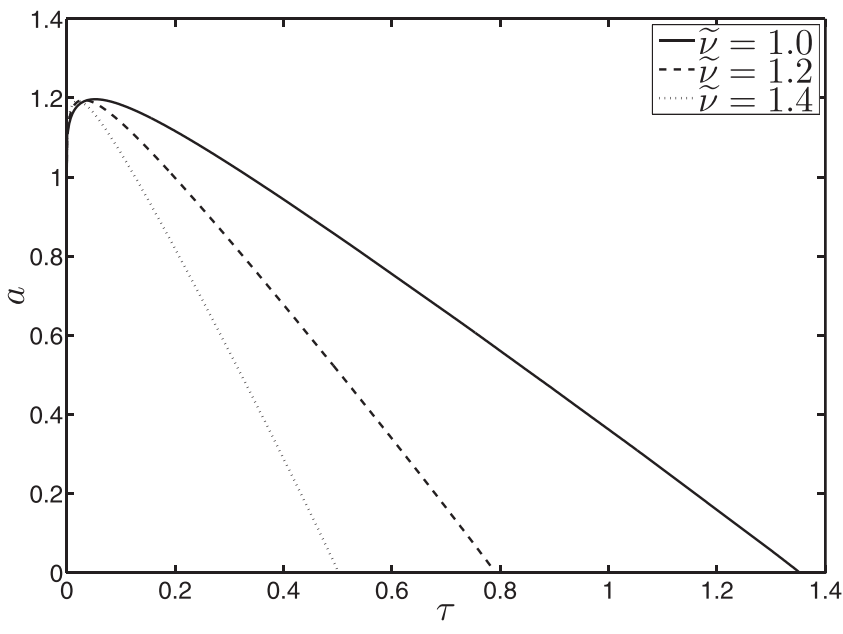

FIG. 10. Amplitude evolution of holes in the absence of drag collisions. These simulations are performed with the critical slope, $\rho(\dot{s})=\rho_{c}$.

separatrix expansion. Accordingly, the lifetime of holes in the presence of diffusion, shown in Figure 12, is found to conform with the expected analytical scaling $\tau_{L} \propto \tilde{\nu}^{-3} .9$ In contrast to holes, calculations of clump lifetimes would, in general, require a generalization of our present model to the case of more than one minimum within the spatial period of the wave. As noted in Ref. 12, additional minima in $y(\xi)$ appear at $\xi=0$ and 1 when the wave phase velocity subsides below $\dot{s}_{0} / 2$. These minima form their own regions with locally trapped particles, which we deliberately refrain from considering here in order to simplify our numerical procedure. Instead, we use the single-minimum model that still applies to clumps when the rate of diffusion is sufficiently large (so that the clumps decay before additional minima have time to develop). The clump amplitudes in Figure 11 correspond to simulations of this type, performed with the constant slope $\rho(\dot{s})=1$. On the other hand, the constant slope is known to cause reduction of the wave amplitude over time, even without diffusive collisions. ${ }^{12}$ As a result, the clump lifetime in Figure 12 deviates from the purely diffusive scaling $\tau_{L} \propto \tilde{\nu}^{-3}$. For large $\tilde{\nu}$, however, diffusion plays the dominant role, and $\tau_{L}$ tends asymptotically to the

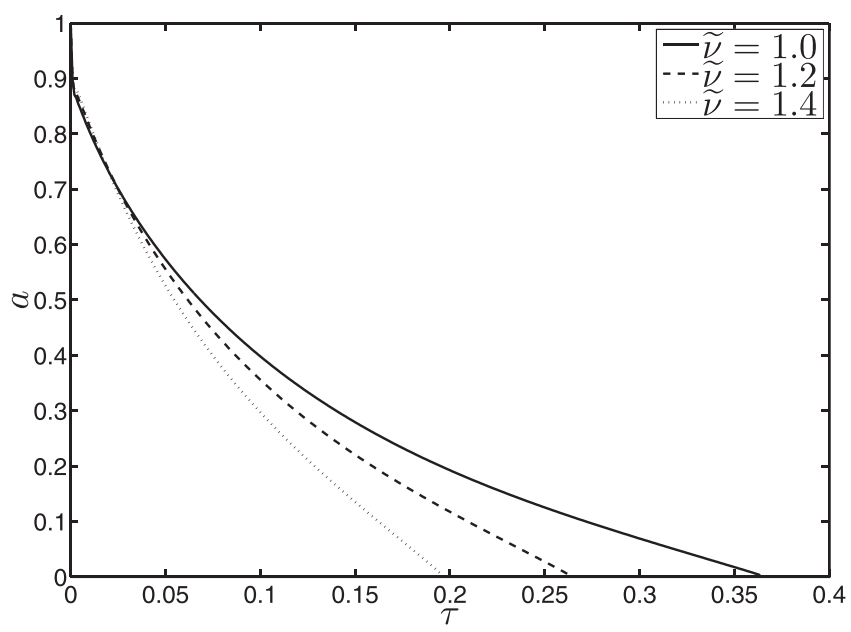

FIG. 11. Amplitude evolution of clumps in the absence of drag collisions. These simulations are run with a constant slope, $\rho(\dot{s})=1$.

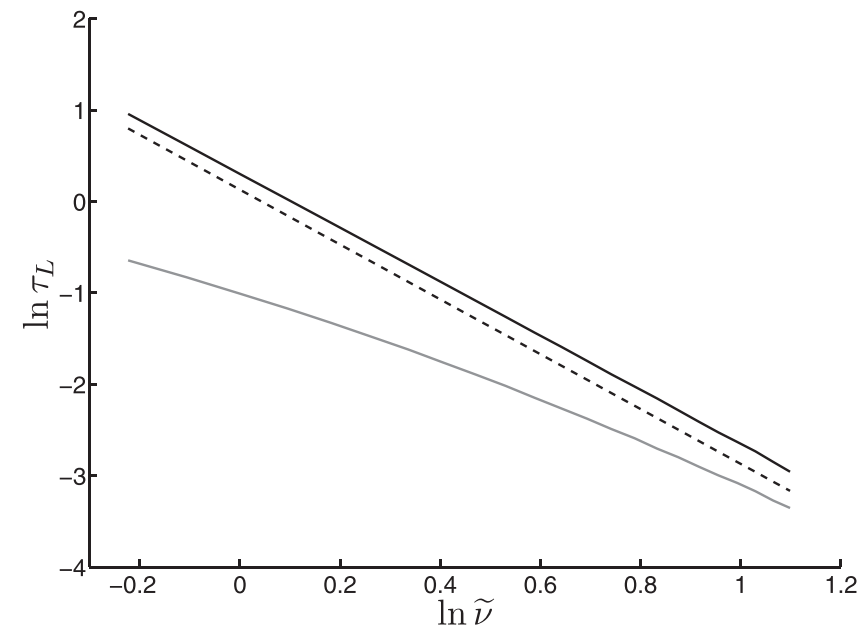

FIG. 12. Lifetimes of holes and clumps as functions of $\tilde{\nu}$, in $\log$-log scale. The solid lines are holes (black line) and clumps (gray line), and the dashed line is the previous theoretical estimate $\tau_{L} \propto \tilde{\nu}^{-3}$.

inverse cubic scaling. The composite distribution used in these simulations of holes and clumps is shown in Figure 13.

\section{Interplay of drag and diffusion}

Non-monotonic frequency sweeping of holes may arise in the presence of a drag term in the fast particle collision operator, ${ }^{9}$ i.e., when $\tilde{\alpha} \neq 0$. The turning points, at which the sweep reverses, are found when $\tilde{\alpha}^{2}$ on the left hand side of Eq. (10c) balances the right hand side, so that $d \dot{s} / d \tau$ vanishes. Figure 14 shows in detail the transition from monotonic to non-monotonic (so called hooked) sweeping pattern, when the drag collision frequency is set to $\tilde{\alpha}=1$ and the diffusion collision frequency $\tilde{\nu}$ varies from 2.2 to 2.5 . The hooks are shown for $\tilde{\nu} \geq 2.4$, but the actual transition occurs at $\tilde{\nu}=2.33$. The mode amplitudes, shown in Figure 15, also exhibit a rollover for $\tilde{\nu} \geq 2.33$, eventually decreasing to zero as the holes disappear and the frequency sweeps terminate. In order to connect to previous studies, ${ }^{9,13}$ these simulations are performed with the constant slope $\rho(\dot{s})=1$.

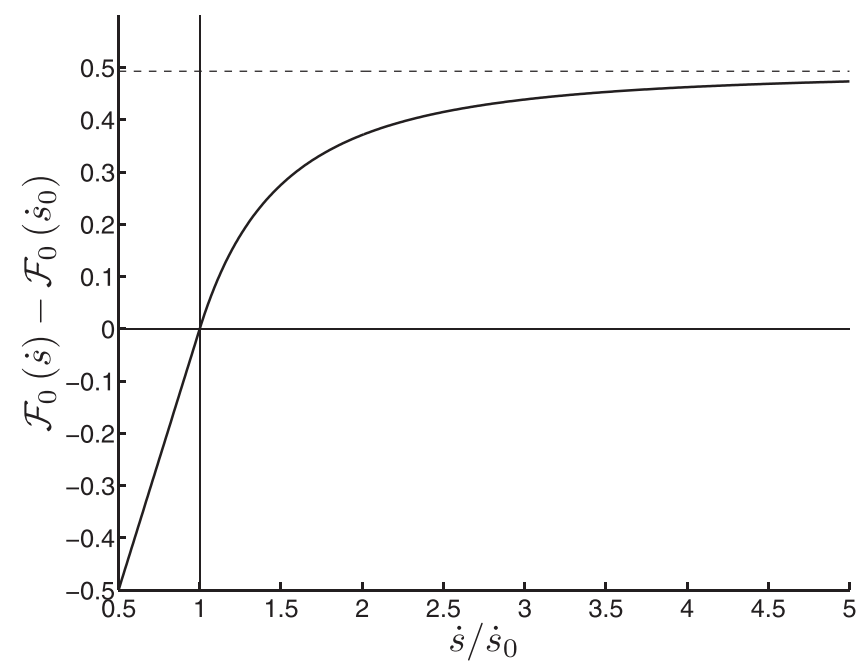

FIG. 13. Unperturbed fast particle distribution function, $\mathcal{F}_{0}(\dot{s})$, corresponding to $\rho(\dot{s})=\rho_{c}$ for $\dot{s}>\dot{s}_{0}$ and $\rho(\dot{s})=1$ for $\dot{s}<\dot{s}_{0} . \mathcal{F}_{0}$ tends asymptotically to $3(2 / \pi)^{4} \approx 0.49$ for large $\dot{s}$. 


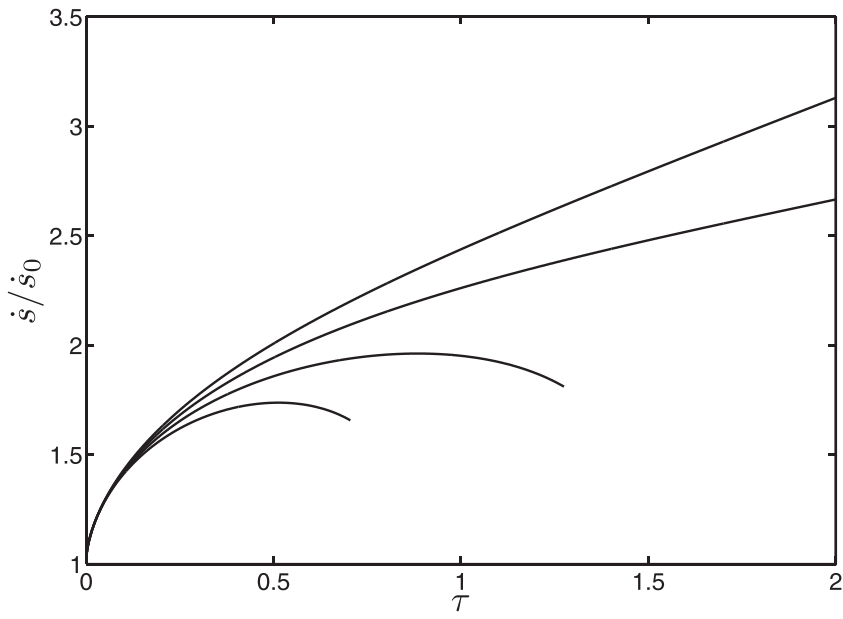

FIG. 14. Frequency evolution of holes at the threshold between monotonic and hooked non-monotonic sweeping in a constant equilibrium slope. The collision frequency for drag is set to $\tilde{\alpha}=1$ and $\tilde{\nu}$ equals, from the top to bottom curve, $2.2,2.3,2.4,2.5$. The transition occurs at $\tilde{\nu}=2.33$.

As shown in Figure 16, hooked frequency sweeping appears for all values of $\tilde{\alpha}$, once $\tilde{\nu}$ exceeds a certain threshold value. The corresponding transition line in $(\tilde{\alpha}, \tilde{\nu})$-space also serves as a boundary of the domain in which phase space holes decay due to diffusive collisions. The supplementary domain exhibits either asymptotically steady holes or holes that sweep indefinitely through the linear distribution of ambient particles.

Asymptotically steady holes emerge when $\tilde{\alpha}$ exceeds a bifurcation threshold value of 2.41. The relaxation to steady state is either monotonic or oscillatory. The number and magnitudes of the oscillations increase with $\tilde{\nu}$, for both $\dot{s}$ and $a$, whereas their steady state values decrease with $\tilde{\nu}$. Figure 17 displays a series of spectral lines that demonstrate this trend for $\tilde{\alpha}=3.5$. As seen in Figure 18, the corresponding amplitudes behave similarly. The steady holes exhibit a characteristic linear $E$-space profile, maintained through the interplay of drag and diffusion, cf., Figure 19. Steady holes are discussed further in Sec. V.

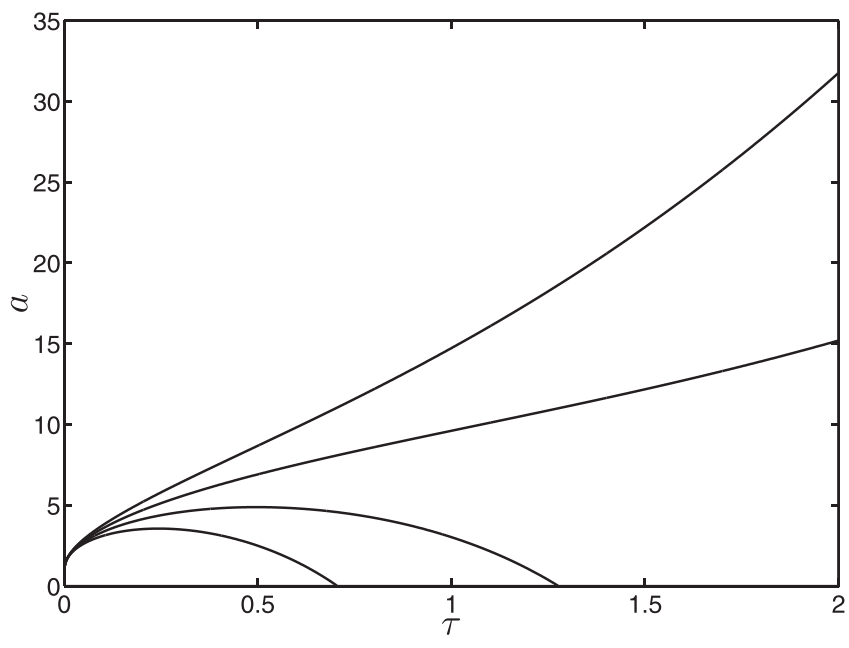

FIG. 15. Amplitude evolution corresponding to the curves in Figure 14.

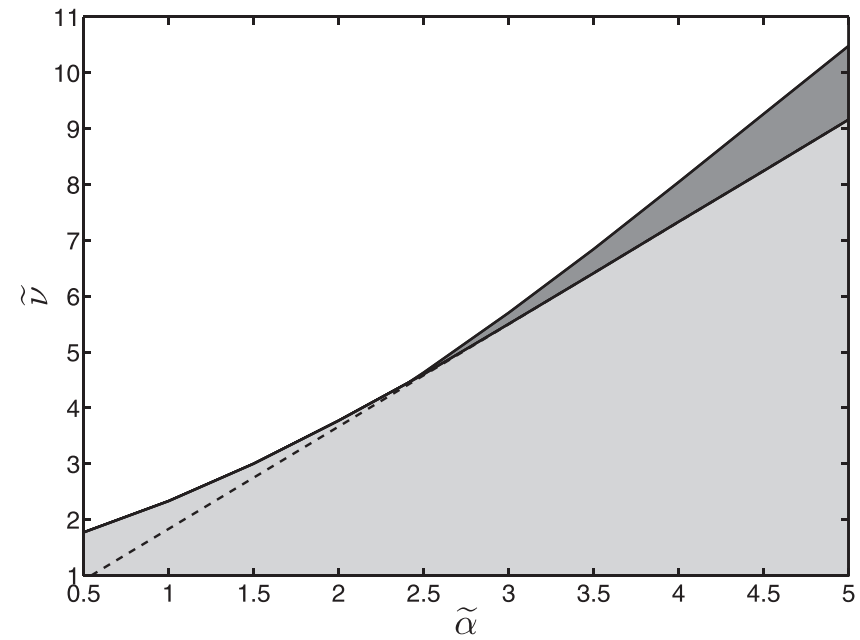

FIG. 16. Parameter domains in $(\tilde{\alpha}, \tilde{\nu})$-space for the three classes of solutions: Monotonically increasing frequency and amplitude (gray area), hooked non-monotonic regime (blank area) and asymptotic steady states (dark area). The dashed, straight line is an approximate analytical boundary of the steady state domain, given by Eq. (44).

\section{STEADY HOLES}

The trapped particle distribution inside a steady hole is given by the stationary solution

$$
\delta \mathcal{F}=\frac{2 a \tilde{\alpha}^{2} \rho}{\tilde{\nu}^{3}}[E-1]
$$

of the kinetic equation (10a) with boundary condition $\delta \mathcal{F}(E=1 ; \tau)=0$. As shown in Figure 19, this expression matches the asymptotic results of numerical simulations with $\rho(\dot{s})=1$ to within $0.1 \%$. It is noteworthy that the linear profile (29) renders a simple analytic description of steady state holes. With Eq. (29), evaluation of the integrals on the right hand side of Eq. (10b) and in $C_{2}$ gives

$$
\begin{aligned}
\frac{\partial^{2} y}{\partial \xi^{2}}+4 \pi^{2}\left(\frac{\dot{s}_{0}}{\dot{s}}\right)^{2} y= & \frac{4 \pi^{3} \tilde{\alpha}^{2} \rho \sqrt{a}}{\tilde{\nu}^{3}}\left[(1-y)^{3 / 2}-\frac{2}{5}\right] \\
& +2 \pi^{2}\left(\frac{\dot{s}_{0}}{\dot{s}}\right)^{2},
\end{aligned}
$$

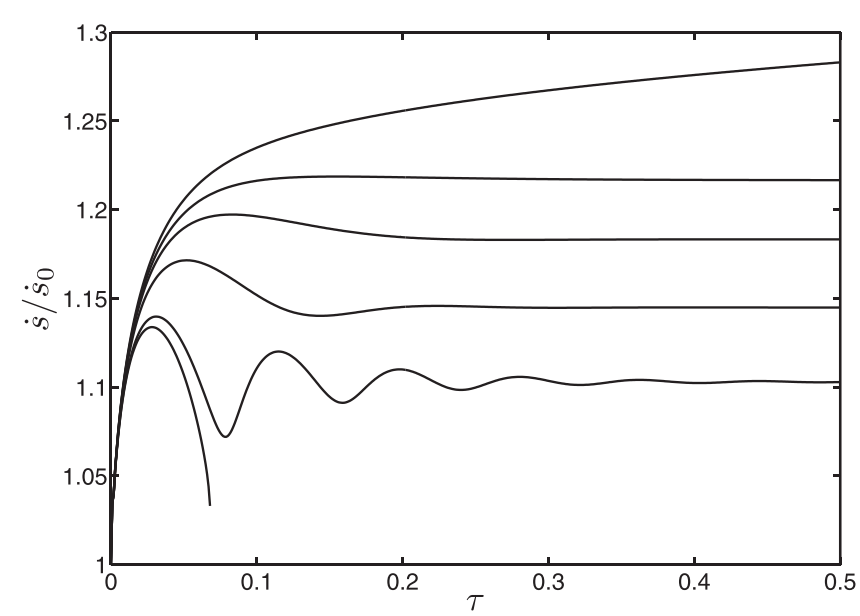

FIG. 17. Frequency evolution of holes when $\tilde{\alpha}=3.5$ and $\tilde{\nu}$ varies across the steady state domain in Figure 16. The monotonically increasing spectral line has $\tilde{\nu}=6.4$, the steady states have, from top to bottom, $\tilde{\nu}=6.45,6.5,6.6$, 6.8 , and the hook has $\tilde{\nu}=6.85$. 


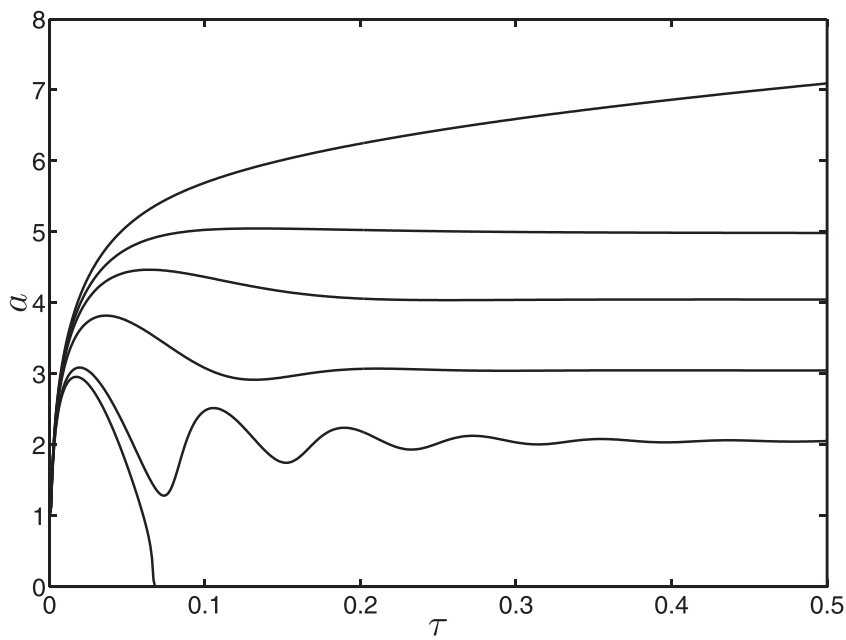

FIG. 18. Amplitude evolution corresponding to the spectral lines in Figure 17.

where $\dot{s}, a$, and $y$ now represent steady state values. The first integral of Eq. (30) can be conveniently written by defining

$$
g=\sqrt{1-y},
$$

which yields

$$
\left(\frac{\partial g}{\partial \xi}\right)^{2}=\pi^{2}\left(\frac{\dot{s}_{0}}{\dot{s}}\right)^{2}\left(1-g^{2}\right)+\frac{4 \pi^{3} \tilde{\alpha}^{2} \rho \sqrt{a}}{5 \tilde{\nu}^{3}}\left(1-g^{3}\right) .
$$

Equation (32) is separable and can be immediately integrated to give an expression for $\xi$ as a function of $g$. The wave profile is then obtained by inverting this relation and forming $y=1-g^{2}$. However, the inversion involves evaluation of elliptic integrals. An equivalent, but more straightforward, approach is to construct a numerical solution for $g$ by first differentiating Eq. (32) with respect to $\xi$ to obtain a harmonic oscillator equation with quadratic nonlinearity,

$$
\frac{\partial^{2} g}{\partial \xi^{2}}+\pi^{2}\left(\frac{\dot{s}_{0}}{\dot{s}}\right)^{2} g=-A g^{2}, \quad A \equiv \frac{6 \pi^{3} \tilde{\alpha}^{2} \rho \sqrt{a}}{5 \tilde{\nu}^{3}} .
$$

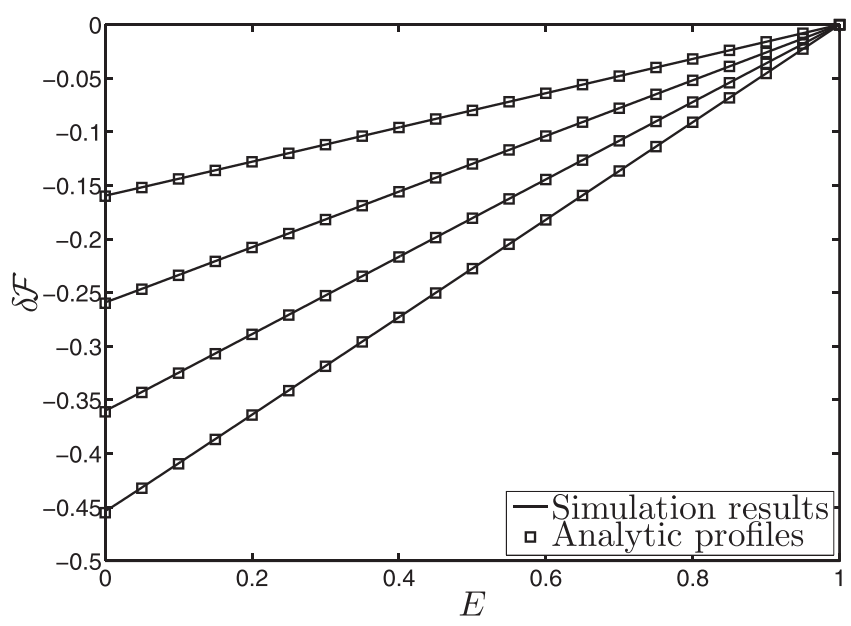

FIG. 19. Steady state $E$-space profiles for the steady holes in Figures 17 and 18. From bottom to top, these holes have $\tilde{\nu}=6.45,6.5,6.6,6.8$. The squares are analytic steady state profiles given by Eq. (29).

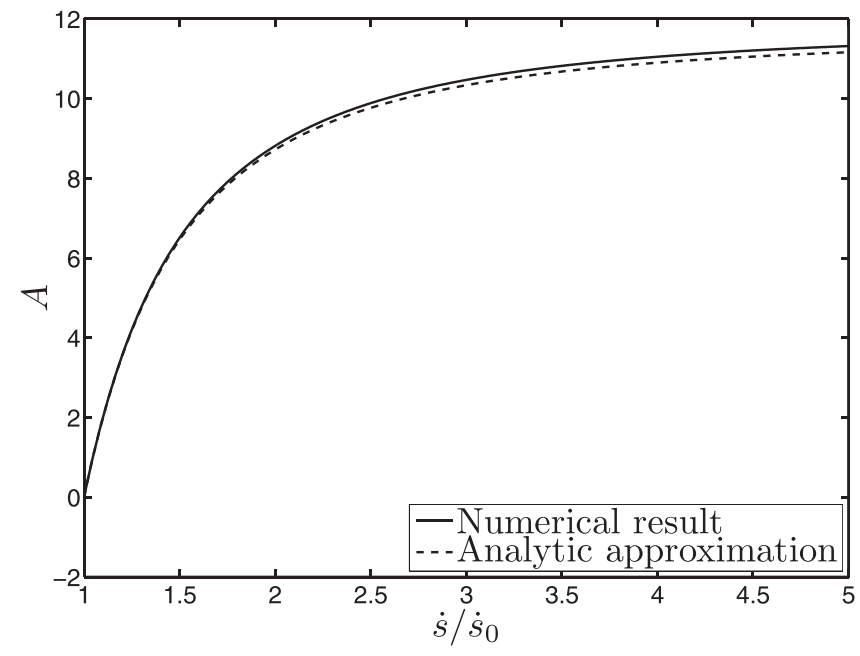

FIG. 20. Eigenvalues $A=A(\dot{s})$ of the nonlinear harmonic oscillator (33).

The boundary conditions for $g$ are $g=1$ and $\partial g / \partial \xi=0$ at $\xi=0.5$ and $g=0$ at $\xi=0,1$. Equation (33) represents a differential "eigenvalue" problem: Given a value of $\dot{s}, A$ is found by shooting from $\xi=0.5$ until $g$ vanishes at $\xi=1$. The relation $A=A(\dot{s})$ found through this procedure is shown in Figure 20.

A remarkable feature of the nonlinear differential equation (33) is that the steady state mode structure never deviates significantly from $g_{0}(\xi)=\sin \pi \xi$, formally obtained from Eq. (33) in the limit $\dot{s} \rightarrow \dot{s}_{0}, A \rightarrow 0$. This observation was first made during the simulations presented in Sec. IV and then confirmed by numerical solutions of Eq. (33). Analytically, it can be seen by rewriting Eq. (33) as

$$
\frac{\partial^{2} g}{\partial \xi^{2}}+\pi^{2} g=\pi^{2} \frac{\dot{s}^{2}-\dot{s}_{0}^{2}}{\dot{s}^{2}} g-A g^{2},
$$

multiplying through with $g_{0}$ and integrating from $\xi=0$ to 1 . We then have

$$
A \int_{0}^{1} g^{2}(\xi) \sin \pi \xi d \xi=\pi^{2} \frac{\dot{s}^{2}-\dot{s}_{0}^{2}}{\dot{s}^{2}} \int_{0}^{1} g(\xi) \sin \pi \xi d \xi .
$$

Assuming that $g \approx g_{0}$, which should hold at least for $\left(\dot{s}-\dot{s}_{0}\right) / \dot{s}_{0} \ll 1, a \ll 1$, gives

$$
A=\frac{6 \pi^{3} \tilde{\alpha}^{2} \rho \sqrt{a}}{5 \tilde{\nu}^{3}} \approx \frac{3 \pi^{3}}{8} \frac{\dot{s}^{2}-\dot{s}_{0}^{2}}{\dot{s}^{2}}
$$

so that

$$
a \approx \frac{25}{256} \frac{\tilde{\nu}^{6}}{\tilde{\alpha}^{4}}\left(\frac{\dot{s}^{2}-\dot{s}_{0}^{2}}{\rho \dot{s}^{2}}\right)^{2}
$$

The approximation (36) is plotted as a dashed curve alongside the numerically obtained relation $A=A(\dot{s})$ in Figure 20. The good agreement between the two lines, even for large $\dot{s} / \dot{s}_{0}$, confirms the closeness of $g$ to $g_{0}$.

A second relation between $\dot{s}$ and $a$, needed in order to close the system, is provided by the power balance condition 
(10c). In steady state, the dissipation in the background plasma is balanced solely by the ambient particles that skim the separatrix due to drag-type collisions. The power balance then reads

$$
\tilde{\alpha}^{2}=\frac{\gamma_{d}}{\gamma_{L 0}} \frac{1}{\sqrt{2} C_{1}}\left(\frac{\dot{s}_{0}}{\dot{s}}\right)^{3} \int_{0}^{1}\left[2 a y(\xi)+\mathcal{U}_{\min }\right]^{2} d \xi,
$$

where now

$$
C_{1}=\frac{8 \sqrt{2} \tilde{\alpha}^{2} \rho a^{3 / 2}}{15 \tilde{\nu}^{3}}-\frac{2 \sqrt{2}}{3 \pi}\left(\frac{\dot{s}_{0}}{\dot{s}}\right)^{2}\left[a+\mathcal{U}_{\min }\right]
$$

and $\mathcal{U}_{\text {min }}$ is determined through Eq. (13). Taken together, Eqs. (33) and (38) determine $\dot{s}$ and $a$ for a given set of input parameters $\gamma_{d} / \gamma_{L 0}, \tilde{\alpha}, \tilde{\nu}$, and $\rho$. However, Eq. (38) also simplifies considerably for $g \approx g_{0}$. We then have $\mathcal{U}_{\min }=-a$, so that the power balance becomes

$$
\tilde{\alpha}^{2} \approx \frac{\gamma_{d}}{\gamma_{L 0}} \frac{15 \tilde{\nu}^{3} \sqrt{a}}{32 \tilde{\alpha}^{2} \rho}\left(\frac{\dot{s}_{0}}{\dot{s}}\right)^{3} .
$$

This handy formula and Eq. (37) give an algebraic equation

$$
\frac{\gamma_{d}}{\gamma_{L 0}} \frac{75}{512}\left(\frac{\tilde{\nu}}{\tilde{\alpha}}\right)^{6} \frac{\left(\dot{s} / \dot{s}_{0}\right)^{2}-1}{\rho^{2}\left(\dot{s} / \dot{s}_{0}\right)^{5}} \approx 1
$$

for the asymptotic value of $\dot{s}$.

The presented solution can be applied to the steady holes found in the simulations in Sec. IV by setting $\rho(\dot{s})=1$ and $\gamma_{d} / \gamma_{L 0}=1$. We then find that for a given value of $\tilde{\alpha}$, there is a critical value of $\tilde{\nu}$ below which no steady states exist. This is visualized in Figure 21, showing plots of the approximate "residual"

$$
R=\frac{75}{512}\left(\frac{\tilde{\nu}}{\tilde{\alpha}}\right)^{6} \frac{\left(\dot{s} / \dot{s}_{0}\right)^{2}-1}{\left(\dot{s} / \dot{s}_{0}\right)^{5}}-1 .
$$

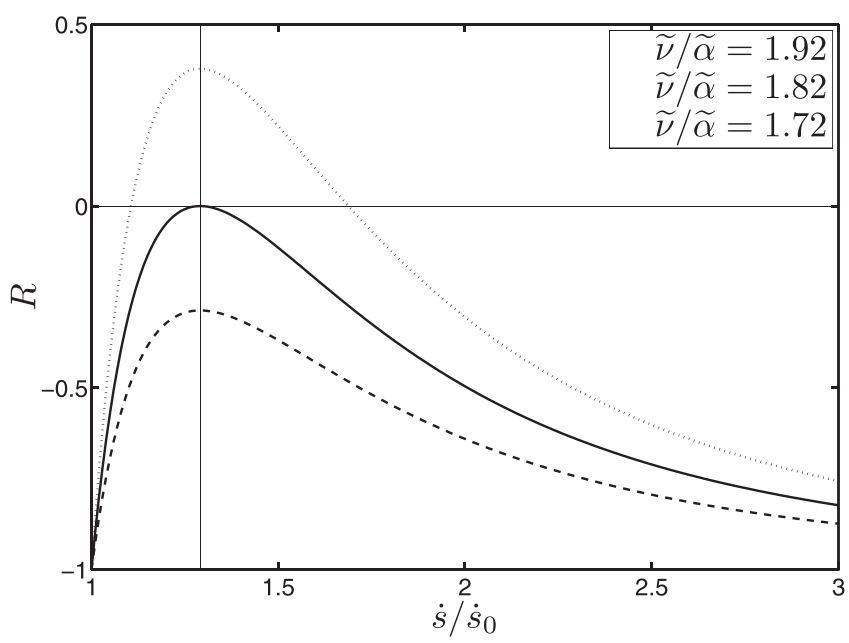

FIG. 21. The approximate power balance residual (42) for $\gamma_{d} / \gamma_{L 0}=1$ and $\rho(\dot{s})=1$. The curves start at and asymptote to -1 , with a maximum at $\dot{s} / \dot{s}_{0}=\sqrt{5 / 3} \approx 1.29$. As $\tilde{\nu} / \tilde{\alpha}$ increases the curves are stretched upwards, with solutions to $R=0$ emerging at $\tilde{\nu} / \tilde{\alpha} \approx 1.82$.
We note that $R$ is always negative for

$$
\frac{\tilde{\nu}}{\tilde{\alpha}}<\left(\frac{256}{9} \sqrt{\frac{5}{3}}\right)^{1 / 6} \approx 1.82
$$

in which case no steady states exist, whereas higher values of $\tilde{\nu} / \tilde{\alpha}$ yield two roots that shift away from the maximum at $\dot{s} / \dot{s}_{0}=\sqrt{5 / 3} \approx 1.29$ as $\tilde{\nu} / \tilde{\alpha}$ increases. The corresponding straight line, given by

$$
\tilde{\nu}=\left(\frac{256}{9} \sqrt{\frac{5}{3}}\right)^{1 / 6} \tilde{\alpha},
$$

is very close to the lower transition line in Figure 16 for $\tilde{\alpha} \gtrsim 2.5$. The exact residual curves for Eq. (38) are very similar to Eq. (42), with maxima at $\dot{s} / \dot{s}_{0}=1.3$. When steady states do exist, the corresponding lower roots reproduce with high accuracy the asymptotic values of $\dot{s}$ and $a$ found in the simulations, cf., Figures 22 and 23. However, no steady holes corresponding to upper roots were found in the simulations. The steady state values of $\dot{s}$ are therefore bounded to $\dot{s} / \dot{s}_{0} \in(1,1.3]$.

The absence of "upper root holes" in the simulations suggests that such solutions are likely to be unstable and decay in a manner similar to the hooks. This calls for stability analysis to clarify whether or not hooks represent unstable steady state solutions, as suggested in Ref. 9. Numerically, a steady hole near the upper transition line in Figure 16 can be slightly pushed into the hook domain via a small increase in $\tilde{\nu}$. Along the transition line, this procedure induces perturbations in $\dot{s}, a$, and $\delta \mathcal{F}$ that grow in an oscillatory manner, thus supporting the conjecture that the upper transition line marks the stability boundary.

\section{DISCUSSION}

The presented numerical simulations of long-range frequency sweeping phenomena extend our earlier adiabatic description of resonant particles in an evolving nonlinear

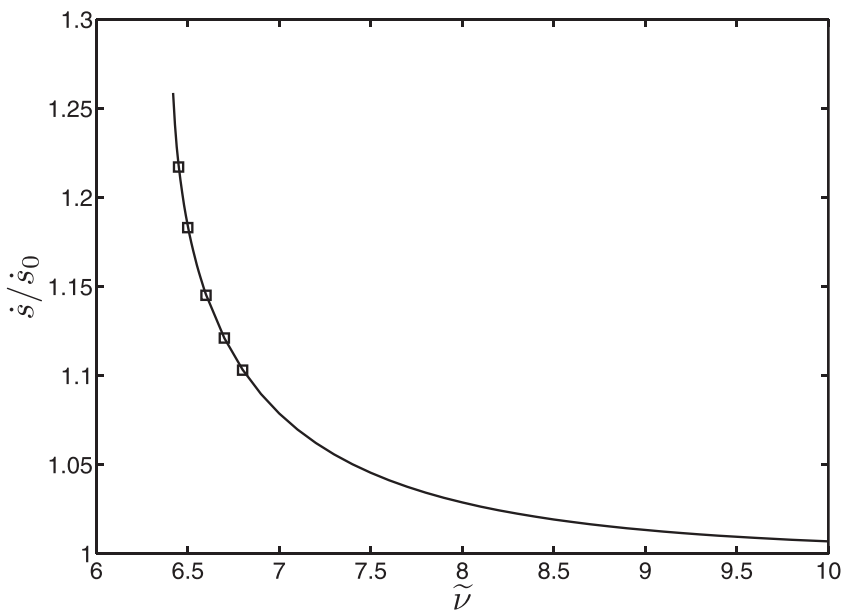

FIG. 22. Steady state frequency as function of $\tilde{\nu}$ when $\tilde{\alpha}=3.5$. The end values in Figure 17 are shown as squares for comparison. 


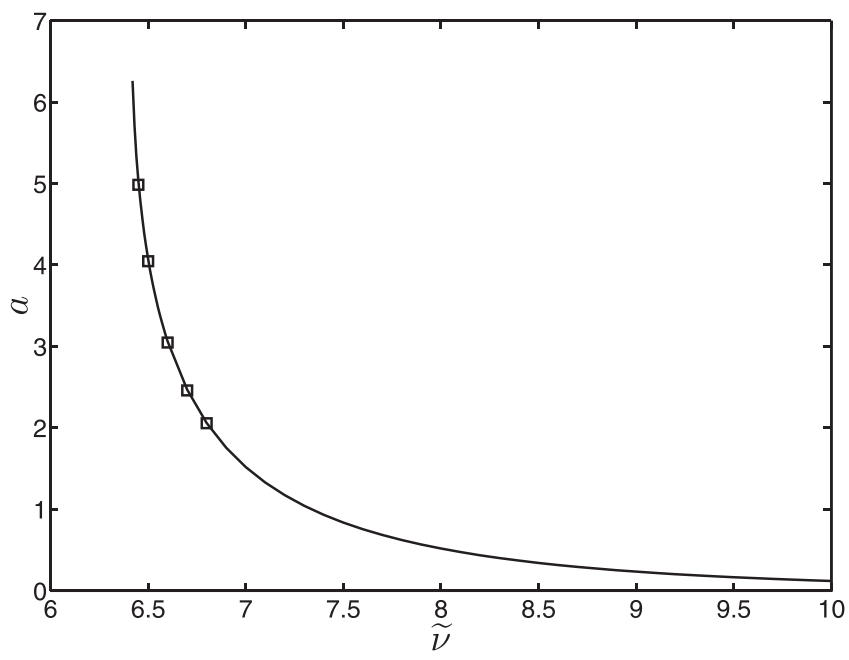

FIG. 23. Steady state amplitude as function of $\tilde{\nu}$ when $\tilde{\alpha}=3.5$. The end values in Figure 18 are shown as squares for comparison.

mode to the regime in which capturing of ambient particles may significantly modify the trapped particle distribution. A convenient feature of the model is that it reduces the problem to the description of trapped particles only, whereas the passing particles can be accounted for via a simple boundary condition at the phase-space separatrix. We observe that the frequency sweeping rate can increase significantly due to particle trapping (as a combined effect of separatrix expansion and diffusive flux trough the separatrix) and that lifetimes of holes and clumps decrease with increasing diffusivity. We have also included drag-type collisions in the simulations and examined sweeping scenarios produced through the interplay between drag and diffusion. The possible types of mode evolution are: 1) persisting sweeping limited only by the range of the fast particle distribution (consequently, the lifetime of these structures should depend on global properties of the equilibrium distribution); 2) establishment of steady nonlinear phase-space structures represented by the newly constructed asymptotic analytic solutions; 3) hook-type pulses with sweep reversal. The third regime suggests recurrent pulses of hooks in the presence of a continuously acting fast particle source. We also note that, if not initiated properly, the phase space structures can exhibit fast transient behavior before they meet the applicability conditions for the adiabatic description.

The spectral asymmetry observed in Figure 9, where holes sweep significantly longer than clumps, is due to the global shape of the unperturbed distribution shown in Figure 13, chosen just to highlight the effect of diffusion on the mode evolution. Other types of distributions can alter the sweeping pattern. In particular, setting $\rho(\dot{s})=\rho_{c}$ for all $\dot{s} \geq$ 0 produces almost perfectly symmetric sweeps (until additional minima appear in $y(\xi ; \tau)$ for the clumps, as discussed in Sec. IV B). In the present 1D model, there are several additional means of generating up/down asymmetries. First and foremost, the considered drag collision operator acts intrinsically asymmetrically to always deepen holes and deprive clumps, while boosting clump sweeping rates and restraining those of holes. ${ }^{13}$ Moreover, the collision rates $\tilde{\alpha}, \tilde{\beta}$, and $\tilde{\nu}$ in general depend on $\dot{s}$, as does the phenomenological linear damping rate $\gamma_{d}$. This opens up an interesting question of whether the listed factors are sufficient to understand the asymmetries of frequency shifts observed in several experiments, including, e.g., TAE avalanches on NSTX.,3

Looking ahead, we consider this work a step towards reduced modeling of frequency sweeping phenomena in tokamak experiments, where the observed signals are likely to be associated with energetic particles locked in isolated nonlinear resonances. The next technical step in this direction is to extend the presented formalism to Alfvénic perturbations, including a relevant description of the fast ion orbits in realistic geometry, as outlined in Ref. 12. This extension should also include diffusive collisions and drag, which now seems feasible with the numerical procedure described in this paper. A more challenging aspect of the realistic modeling is to develop a proper description of continuum damping for Alfvén waves. The subtlety here is that continuum resonances themselves may require nonlinear treatment, which is not captured by our present model with a preset background damping rate.

\section{ACKNOWLEDGMENTS}

The authors are grateful to M. K. Lilley and F. Håkansson for useful comments and to M. Lisak for his support that made this collaboration possible. The work was funded jointly by the Swedish Research Council, EURATOM, and the U.S. Department of Energy Contract No. DE-FG02-04ER54742.

${ }^{1}$ D. Maslovsky, B. Levitt, and M. E. Mauel, Phys. Rev. Lett. 90(18), 185001 (2003).

${ }^{2}$ E. Fredrickson, N. Gorelenkov, R. Bell, J. Menard, A. Roquemore, S. Kubota, N. Crocker, and W. Peebles, Nucl. Fusion 46, S926 (2006).

${ }^{3}$ E. Fredrickson, R. E. Bell, D. S. Darrow, G. Y. Fu, and N. N. Gorelenkov, Phys. Plasmas 13, 056109 (2006).

${ }^{4}$ S. D. Pinches, H. L. Berk, M. P. Gryaznevich, S. E. Sharapov, and JET-EFDA Contributors, Plasma Phys. Controlled Fusion 46, S47 (2004).

${ }^{5}$ M. P. Gryaznevich and S. E. Sharapov, Nucl. Fusion 46, S942 (2006).

${ }^{6}$ H. L. Berk, B. N. Breizman, and N. V. Petviashvili, Phys. Lett. A 234, 213 (1997).

${ }^{7}$ B. N. Breizman, H. L. Berk, M. S. Pekker, F. Porcelli, G. V. Stupakov, and K. L. Wong, Phys. Plasmas 4(5), 1559 (1997).

${ }^{8}$ H. L. Berk, B. N. Breizman, J. Candy, M. Pekker, and N. V. Petviashvili, Phys. Plasmas 6(8), 3102 (1999).

${ }^{9}$ M. K. Lilley, B. N. Breizman, and S. E. Sharapov, Phys. Plasmas 17, 092305 (2010).

${ }^{10}$ C. T. Hsu, C. Z. Cheng, P. Helander, D. J. Sigmar, and R. White, Phys. Rev. Lett. 72(16), 2503 (1994).

${ }^{11}$ M. K. Lilley and B. N. Breizman, Nucl. Fusion 52, 094002 (2012).

${ }^{12}$ B. N. Breizman, Nucl. Fusion 50, 084014 (2010).

${ }^{13}$ R. M. Nyqvist, M. K. Lilley, and B. N. Breizman, Nucl. Fusion 52, 094020 (2012).

${ }^{14}$ A. V. Timofeev, Sov. Phys. JETP 48(4), 656 (1978).

${ }^{15}$ J. L. Tennyson, J. R. Cary, and D. F. Escande, Phys. Rev. Lett. 56(20), 2117 (1986)

${ }^{16}$ J. R. Cary, D. F. Escande, and J. L. Tennyson, Phys. Rev. A 34(5), 4256 (1986).

${ }^{17}$ M. K. Lilley, B. N. Breizman, and S. E. Sharapov, Phys. Rev. Lett. 102, 195003 (2009).

${ }^{18}$ D. Yu. Eremin and H. L. Berk, Phys. Plasmas 9(3), 772 (2002). 